\title{
Improved photocatalytic activity of anatase titanium dioxide by reduced graphene oxide
}

\author{
Nor Shuhada Alim ${ }^{1}$, Hendrik O. Lintang ${ }^{2}$, Leny Yuliati ${ }^{2 *}$ \\ ${ }^{1}$ Department of Chemistry, Faculty of Science, Universiti Teknologi Malaysia, 81310 UTM Johor Bahru, Johor, Malaysia. \\ ${ }^{2}$ Centre for Sustainable Nanomaterials, Ibnu Sina Institute for Scientific and Industrial Research, Universiti Teknologi Malaysia, 81310 UTM Johor Bahru, \\ Johor, Malaysia. \\ “Corresponding Author: leny@ibnusina.utm.my
}

\section{Article history :}

Received 31 October 2015

Accepted 13 November 2015

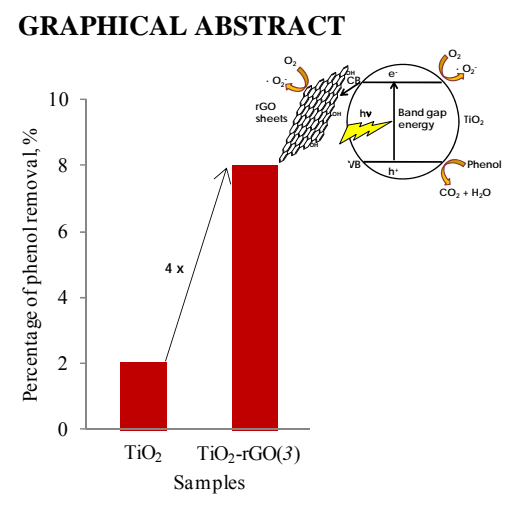

\begin{abstract}
Reduced graphene oxide (rGO) has been one of the most investigated carbon based materials due to its great electronic conductivity that make it able to accept and transport electron easily. In order to study the effect of $\mathrm{rGO}$, anatase titanium dioxide-reduced graphene oxide $\left(\mathrm{TiO}_{2}-\mathrm{rGO}\right)$ composite was prepared by UV-assisted photocatalytic reduction method using the anatase $\mathrm{TiO}_{2}$ as a photocatalyst and various different loadings of graphene oxide (GO). The characterizations of the prepared samples were investigated by X-ray diffractometer (XRD), Fourier transform infrared (FTIR), and fluorescence spectroscopies. The XRD patterns and FTIR spectra confirmed that all the $\mathrm{TiO}_{2}$-rGO composites samples were successfully synthesized without disrupting the structure of the anatase $\mathrm{TiO}_{2}$. Fluorescence spectroscopy revealed the role of the $\mathrm{rGO}$ to reduce the electron-hole recombination on the anatase $\mathrm{TiO}_{2}$. In the photocatalytic removal of phenol, all the $\mathrm{TiO}_{2}-\mathrm{rGO}$ composites showed better photocatalytic activities than the bare anatase $\mathrm{TiO}_{2}$ under UV light irradiation. The activity of the anatase $\mathrm{TiO}_{2}$ was enhanced by more than four times with the addition of the GO with the optimum amount ( $3 \mathrm{wt} \%$ ). It was proposed that the good photocatalytic performance obtained on the composites were caused by the successful suppression of electron-hole recombination by the $\mathrm{rGO}$ on the $\mathrm{TiO}_{2}$.
\end{abstract}

Keywords: anatase titanium dioxide, reduced graphene oxide, titanium dioxide-reduced graphene oxide composite, photocatalyst, removal of phenol

\section{INTRODUCTION}

Titanium (IV) oxide $\left(\mathrm{TiO}_{2}\right)$ is the most attractive photocatalyst due to it is nontoxic, chemically stable, and relatively cheap. The well-known drawback in the use of $\mathrm{TiO}_{2}$ in the photocatalytic process is the fast recombination of electron and hole pairs. It has been reported that the recombination of electron-hole pairs can be inhibited by doping of the $\mathrm{TiO}_{2}$ with the noble metals, such as $\mathrm{Pt}, \mathrm{Au}$, and $\mathrm{Pd}[1,2]$. Another option to reduce the recombination is by making heterojunctions of $\mathrm{TiO}_{2}$, such as, $\mathrm{Bi}_{2} \mathrm{~S}_{3} / \mathrm{TiO}_{2}$, and $\mathrm{CdS} / \mathrm{TiO}_{2}$ [3]. However, the use of $\mathrm{CdS}$ is restricted since it may be leached out of the photocatalyst during the reaction due to the photocorrosion [4].

On the other hand, graphene is a very attractive material because of its unique electronic property, flexible structure, optical property in which it presumes great promises for wide applications. In the recently reported studies, graphene based materials has been shown to have potential ability to increase photocatalytic performance of photocatalyst in degradation of organic pollutants [5-8] and also photocatalytic water splitting [9-13]. Therefore, graphene based material is ideal to modify the $\mathrm{TiO}_{2}$ since it can help not only to increase the capability of the $\mathrm{TiO}_{2}$ to absorb the organic pollutant, but also reduce the recombination of electron-hole pair on the $\mathrm{TiO}_{2}$.

Since graphene oxide (GO) contains abundant of hydrophilic groups such as hydroxyl, carboxyl, and epoxy groups bonded with carbon and other atomic scale defects, they may alter the electrical and optical properties of graphene with low transparency, high resistivity and low charge carrier mobility. Repairing the $\mathrm{sp}^{2}$ structure and reduction of $\mathrm{GO}$ to the reduced graphene oxide (rGO) is necessary to be used for charge shuttling and possible site for catalytic application [9]. Recently, various methods have been reported in reducing the $\mathrm{GO}$, such as chemical reduction, thermal reduction, and hydrothermal reduction $[5,6,10]$. However, there are some limitations to these methods, such as the use of hydrazine that is highly toxic and high temperature that will affect the structure of the formed rGO [10]. Recently, Williams et al. discovered a clean method by using $\mathrm{TiO}_{2}$ as a photocatalyst to convert GO to rGO at room temperature [14]. The advantages of the photocatalytic reduction method are low cost and feasible to form partially reduced graphene oxide, which is favorable for the photocatalytic applications.

In the present study, anatase $\mathrm{TiO}_{2}$ was used as the photocatalyst to prepare the $\mathrm{TiO}_{2}-\mathrm{rGO}$ composites by UV- 
assisted photocatalytic reduction method in the presence of methanol as the reducing agent. The photocatalytic activities of the $\mathrm{TiO}_{2}$-rGO composite samples were assessed by examining the removal of phenol under UV light irradiation.

\section{EXPERIMENTS}

\subsection{Preparation of $\mathrm{TiO}_{2}$-rGO Composites}

GO powder was synthesized via an improved Hummers' method [15]. The commercial graphite powder (Sigma-Aldrich) was reacted with a mixture of $\mathrm{KMnO}_{4}$, $\mathrm{H}_{2} \mathrm{SO}_{4}$ and $\mathrm{H}_{3} \mathrm{PO}_{4}$. The mixture was centrifuged and washed twice with $\mathrm{HCl}$, followed by double distilled water until $\mathrm{pH} 7$ was reached. The obtained solid was dispersed in methanol and ultrasonicated for $1 \mathrm{~h}$. Finally, the solution was evaporated to remove excess solvent and dried at room temperature overnight. The $\mathrm{TiO}_{2} \mathrm{rGO}$ composites were prepared by UV-assisted photocatalytic reduction method [14] with GO contents of $0.5,1,3$, and 5 wt $\%$. The composite samples were labeled as $\mathrm{TiO}_{2}-\mathrm{rGO}(x)$, which $x$ shows the $\mathrm{GO}$ contents. For a typical synthesis of $\mathrm{TiO}_{2}-$ rGO(0.5), anatase $\mathrm{TiO}_{2}$ nanoparticles (1 g, Sigma-Aldrich) and the prepared GO $(0.005 \mathrm{~g})$ was dispersed in methanol $(60 \mathrm{~mL})$ and sonicated for $30 \mathrm{~min}$. After sonication, the mixture was transferred into a $100 \mathrm{~mL}$ jacketed beaker and placed on a stirring plate with a cooling water system to ensure that the reaction could be carried out at room temperature. The synthesis reaction was conducted in a closed box for $24 \mathrm{~h}$ under $8 \mathrm{~W}$ UV light irradiation with wavelength $(\lambda)$ of $254 \mathrm{~nm}$ and intensity $(I)$ of 0.32 $\mathrm{mw} / \mathrm{cm}^{2}$. After each reaction, the obtained solid was filtered and washed with distilled water and methanol consecutively. The final product was then dried at room temperature. Similar procedures were used to prepare the other $\mathrm{TiO}_{2}-\mathrm{rGO}(x)$ composite photocatalysts.

\subsection{Characterizations}

The prepared composite photocatalysts were characterized using different types of instruments. X-ray diffraction (XRD) patterns were obtained on a Bruker D8 diffractometer using a $\mathrm{Cu} \mathrm{K \alpha}$ at $40 \mathrm{kV}$ and $40 \mathrm{~mA}$. Fourier transform infrared (FTIR) spectra were measured at room temperature using a Nicolet iS50 spectroscopy. Fluorescence spectra were recorded at room temperature using an FP-8500 JASCO.

\subsection{Photocatalytic Activities}

For the photocatalytic activity, the removal of phenol was conducted by adding photocatalyst $(0.05 \mathrm{~g})$ in phenol solution $(50 \mathrm{~mL}, 50 \mathrm{ppm})$, which was prepared by using acetonitrile as a solvent. The mixture was placed on a stirring plate in a closed box in the dark condition for 30 minutes to reach the adsorption equilibrium. The mixture was then put under exposure of $8 \mathrm{~W}$ UV light irradiation $(\lambda$
$=254 \mathrm{~nm}, I=0.32 \mathrm{mw} / \mathrm{cm}^{2}$ ) for $3 \mathrm{~h}$. After reaction, the concentration of the solution was measured by a gas chromatography equipped with a flame ionization detector (GC-FID, 7820A, Agilent Technologies) to determine the percentage of phenol removal by using an equation as shown in Equation 1 below.

Percentage of phenol removal $=\left(C_{o}-C_{t}\right) / C_{o} \times 100 \%$

where $C_{o}$ was the initial phenol concentration and $C_{t}$ was the phenol concentration after reaction under $3 \mathrm{~h} \mathrm{UV} \mathrm{light}$ irradiation at room temperature.

\section{RESULTS AND DISCUSSION}

Figure 1 shows the XRD patterns of the GO and the $\mathrm{TiO}_{2}$-rGO composites. As can be seen in Figure 1(a), a weak diffraction peak was observed at a $2 \theta$ value of $10.4^{\circ}$ (002), which attributed to the interplanar distance of 0.80 $\mathrm{nm}$ and was in good agreement with the previous reported literatures [5,15]. This result indicated that the GO was successfully synthesized from oxidation of graphite via the improved Hummers' method. Figure 1(b-f) illustrate the XRD patterns of the $\mathrm{TiO}_{2}$-rGO composite photocatalysts. It was clear that all the diffraction peaks matched the standard data for pure anatase of $\mathrm{TiO}_{2}$ (JCPDS 84-1286). The diffraction peaks identified at $2 \theta$ values of $25.3,37.8,48.0$, $53.9,55.0$, and $62.7^{\circ}$ can be ascribed as (101), (004), (200), (105), (211), and (118) crystal planes of anatase $\mathrm{TiO}_{2}$. Addition of GO did not affect the crystallinity and the structural phases of the $\mathrm{TiO}_{2}$. Notably, the characteristic peak of the GO was disappeared while the rGO could not be detected in the composites, suggesting the low amount or low diffraction intensity of rGO.

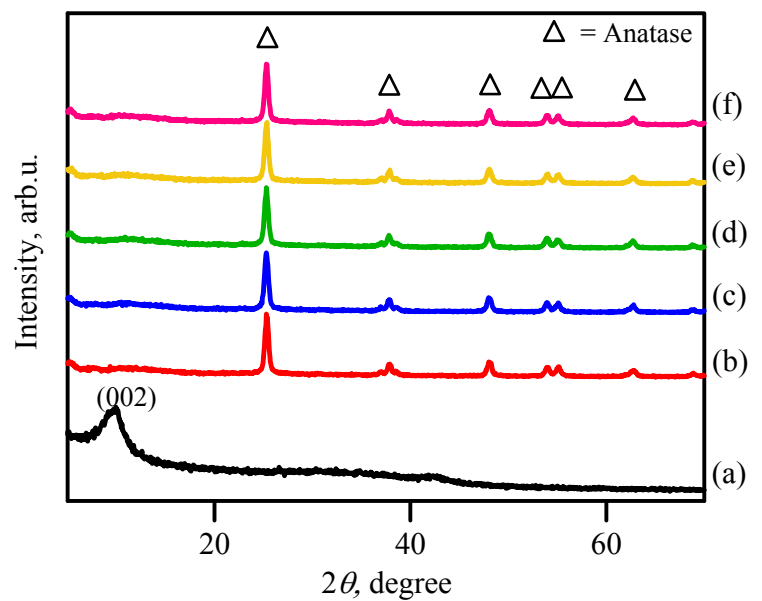

Fig. 1 XRD patterns of (a) $\mathrm{GO}$, (b) $\mathrm{TiO}_{2}$, (c) $\mathrm{TiO}_{2}-\mathrm{rGO}(0.5)$, (d) $\mathrm{TiO}_{2}-\mathrm{rGO}(1)$, (e) $\mathrm{TiO}_{2}-\mathrm{rGO}(3)$, and (f) $\mathrm{TiO}_{2}-\mathrm{rGO}(5)$ samples

Figure 2 illustrates the FTIR spectra of the GO, the $\mathrm{TiO}_{2}$ and the $\mathrm{TiO}_{2}-\mathrm{rGO}$ composites. As can be seen from Figure 2(a), the peak at around $1634 \mathrm{~cm}^{-1}$ was attributed to 
the adsorption of $\mathrm{C}=\mathrm{C}$ aromatic bonding and the broad peak at around 3000 to $3700 \mathrm{~cm}^{-1}$ region was due to physically adsorbed water molecules on the GO [16]. On the other hand, $\mathrm{TiO}_{2}$ exhibited additional broad peaks below $800 \mathrm{~cm}^{-1}$, which corresponded to Ti-O-Ti groups [7]. All the $\mathrm{TiO}_{2}$-rGO composites gave characteristic peaks of both $\mathrm{GO}$ and $\mathrm{TiO}_{2}$, in which the peak at around $1634 \mathrm{~cm}^{-1}$ became more significant with the increase of GO loading, suggesting the presence of $\mathrm{GO}$ in the composite photocatalysts.

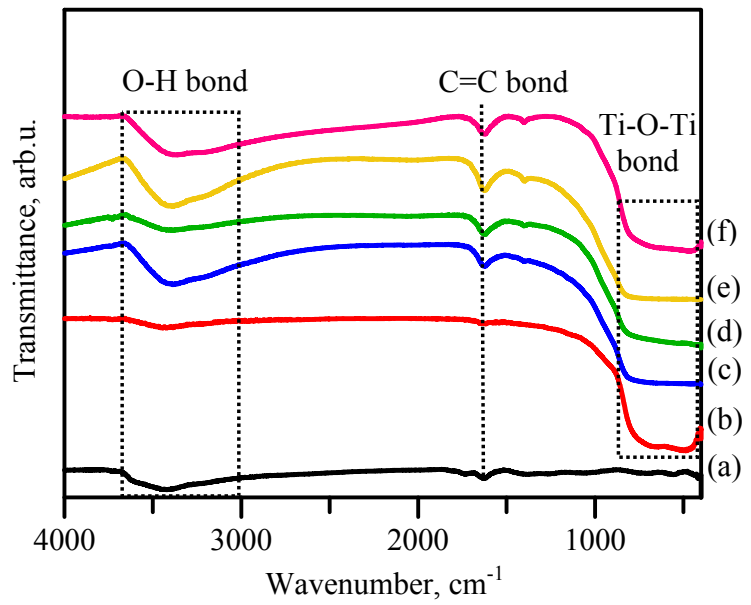

Fig. 2 FTIR spectra of (a) $\mathrm{GO}$, (b) $\mathrm{TiO}_{2}$, (c) $\mathrm{TiO}_{2}-\mathrm{rGO}(0.5)$, (d) $\mathrm{TiO}_{2}-\mathrm{rGO}(1)$, (e) $\mathrm{TiO}_{2}-\mathrm{rGO}(3)$, and (f) $\mathrm{TiO}_{2}-\mathrm{rGO}(5)$ samples

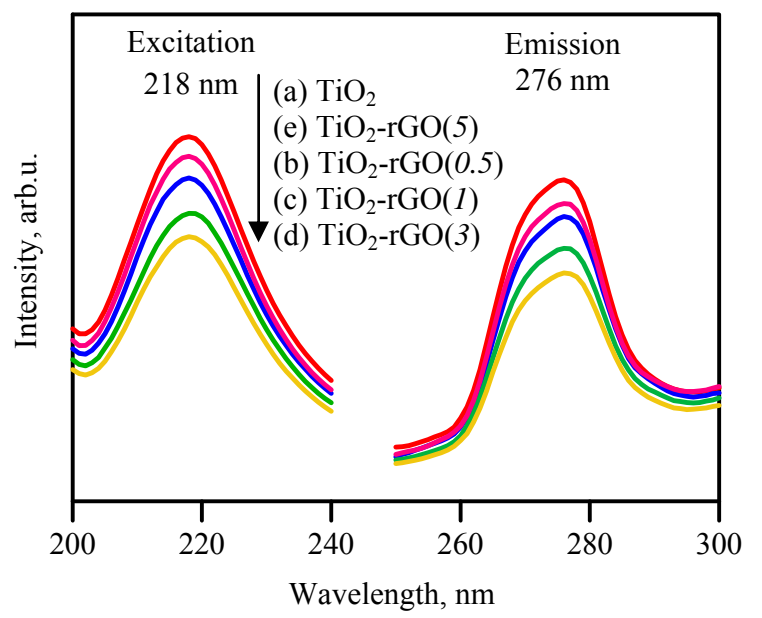

Fig. 3 Fluorescence spectra of (a) $\mathrm{TiO}_{2}$, (b) $\mathrm{TiO}_{2}-\mathrm{rGO}(0.5)$, (c) $\mathrm{TiO}_{2}-\mathrm{rGO}(1)$, (d) $\mathrm{TiO}_{2}-\mathrm{rGO}(3)$, and (e) $\mathrm{TiO}_{2}-\mathrm{rGO}(5)$ samples

The emission sites of $\mathrm{TiO}_{2}$ with different loadings of the GO were studied by fluorescence spectroscopy. Figure 3 illustrates the excitation and emission spectra of $\mathrm{TiO}_{2}$ and $\mathrm{TiO}_{2}$-rGO composite photocatalysts. $\mathrm{TiO}_{2}$ showed strong emission spectrum at $276 \mathrm{~nm}$ with the excitation wavelength of $218 \mathrm{~nm}$. It was confirmed that the addition of the GO did not give any influences on the excitation and emission wavelengths but the intensity of the spectra was found to be affected by the presence of rGO. This suggested that the presence of $\mathrm{rGO}$ might have successfully suppressed the electron-hole recombination of $\mathrm{TiO}_{2}$. It can be observed that the intensity of the excitation and emission spectra decreased with the increasing loading of GO from 0.5 to $3 \mathrm{wt} \%$, while higher loading amount of GO (5 wt\%) gave higher intensity than other $\mathrm{TiO}_{2}-\mathrm{rGO}(x)$ samples. The higher amount of GO might cause the incident of recombination center at $\mathrm{rGO}$, leading to the slight increase in the intensity of excitation and emission spectra [17].

The photocatalytic performances of the $\mathrm{TiO}_{2}-\mathrm{rGO}$ composite photocatalysts were investigated for the phenol removal under $3 \mathrm{~h}$ of $\mathrm{UV}$ light irradiation at room temperature. In order to support that the decrease in the concentration of phenol was contributed solely by the photocatalyst activity, photolysis and adsorption experiments were also conducted. The concentration of phenol remained unchanged after $3 \mathrm{~h}$ of UV light irradiation without photocatalyst. This result suggested that phenol was stable under UV light irradiation, and phenol could not be degraded in the presence of UV light irradiation without the photocatalyst. On the other hand, phenol adsorption test under dark condition showed that adsorption-desorption equilibrium was achieved after 30 min by stirring phenol solution in the presence of photocatalyst. The phenol amount that was adsorbed on the anatase $\mathrm{TiO}_{2}$ photocatalyst was only ca. $1 \%$. This result suggested that phenol could not be degraded in the presence of photocatalyst without UV light irradiation.

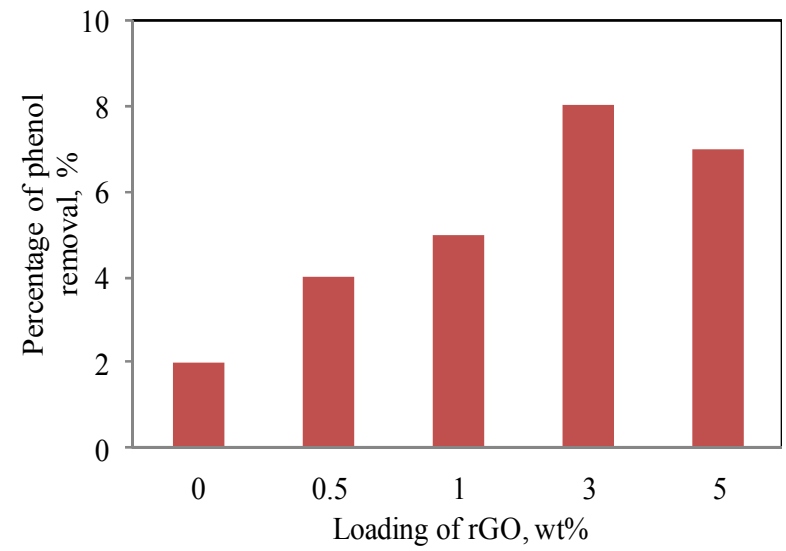

Fig. 4 Percentage of phenol removal on the anatase $\mathrm{TiO}_{2}$ and $\mathrm{TiO}_{2}-\mathrm{rGO}(x)$ composite samples

Figure 4 shows the percentage removal of phenol on the $\mathrm{TiO}_{2}$ and the $\mathrm{TiO}_{2}$-rGO composites with different GO contents. The unmodified $\mathrm{TiO}_{2}$ sample only showed $2 \%$ of phenol removal. It was clear that all the $\mathrm{TiO}_{2}-\mathrm{rGO}$ composites gave higher photocatalytic activity than that of the unmodified $\mathrm{TiO}_{2}$ sample. The activity of the $\mathrm{TiO}_{2}$ sample increased to 4 and $5 \%$ over the composites with addition of 0.5 and $1 \mathrm{wt} \%$ of GO loading, respectively. The photocatalytic activity of $\mathrm{TiO}_{2}$ was further improved by 
almost four times, which was $8 \%$ of phenol removal, when the loading of GO increased to $3 \mathrm{wt} \%$. This good improvement would be due to the presence of rGO in the composite that suppressed the electron-hole recombination of $\mathrm{TiO}_{2}$, as supported by the fluorescence spectra. However, further increase of GO loading to $5 \mathrm{wt} \%$ did not further improve the photocatalytic activity of the $\mathrm{TiO}_{2}$. This result suggested that the optimum loading of $\mathrm{GO}$ onto the $\mathrm{TiO}_{2}$ was $3 \mathrm{wt} \%$. Excess loading of the GO might have caused light scattering, which resulted in a light harvesting competition between $\mathrm{TiO}_{2}$ and $\mathrm{rGO}$, and led to the decrease of the photocatalytic performance [18]. Besides, the excessive rGO can also act as a recombination center that promoted the recombination of electron-hole pair in $\mathrm{rGO}$ and thus, reducing the photocatalytic activity [17].

\section{CONCLUSION}

The $\mathrm{TiO}_{2}-\mathrm{rGO}$ composite photocatalysts were successfully prepared by UV-assisted photocatalytic reduction method. From the characterization, it was revealed that the crystalline structure of $\mathrm{TiO}_{2}$ was not affected with the addition of GO. The presence of $\mathrm{rGO}$ was found to decrease the emission intensity of $\mathrm{TiO}_{2}$, suggesting that the presence of rGO might successfully suppress the electron-hole recombination of $\mathrm{TiO}_{2}$. For the photocatalytic activities, all the prepared samples gave significantly enhanced photocatalytic activity for the phenol removal. The $\mathrm{TiO}_{2}$-rGO sample with $3 \mathrm{wt} \%$ of GO loading exhibited the highest photocatalytic activity, which was 4 times higher than bare anatase $\mathrm{TiO}_{2}$. The efficient suppression of the electron-hole recombination on the $\mathrm{TiO}_{2}$ led to the improvement of the photocatalytic performance.

\section{ACKNOWLEDGEMENTS}

This work has been financially supported by the Ministry of Higher Education (MOHE) and Universiti Teknologi Malaysia (UTM, Malaysia) through the international contract matching grant (cost center code: R.J130000.7626.4C035).

\section{REFERENCES}

[1] S. Kim, S.J. H Wang, W.Y. Choi, J. Phys. Chem. B. 109 (2005) 24260.

[2] N. Sobana, M. Muruganadham, M. Swaminathan, J. Mol. Catal. A: Chem. 258 (2006) 124.

[3] Y. Bessekhouad, D. Robert, J.V. Weber, J. Photochem. Photobiol. A: Chem. 163 (2004) 569.

[4] D.J. Fermin, E.A. Ponomarev, L.M. Peter, J. Electronanal. Chem 473 (1999) 192.

[5] Y. Zhang, Z.R. Tang, X. Fu, Y.J. Xu, ACS Nano. 7303 (2010) 4.

[6] D.P.Sanjaya, G.M Ruperto, V. Khiem, N. Nijem, S. Oliver, C. Yves, J.B. Kenneth, ACS Catal. 2 (2012) 949.

[7] M. Wojtoniszak, B. Zielinska, X. Chen, R.J. Kalenczuk, E. Borowiak-Palen, J. Mater. Sci. 47 (2012) 3187.

[8] R.M. Mohamed. Desalin. Water Treat. 50 (2012) 147.

[9] X. Pan, Y. Zhao, S. Wang, Z. Fan, in: A Méndez-Vilas (Ed). Materials and Processes for Energy: Communicating Current Research and Technological Developments, Formatex Research Center, Spain, 2013, pp. 913-920.

[10] A. Iwase, Y.H. Ng, Y. Ishiguro, A. Kudo, R. Amal, J. Am. Chem Soc. 133 (2011) 11054

[11] W. Fan, Q. Lai, Q. Zhang, Y. Wang. J. Phys. Chem. C. 115 (2011) 10694.

[12] P. Cheng, Z. Yang, H. Wang, W. Chen, M. Chen, S. Wenfeng, G. Ding. Int. J. Hydrogen Energ. 37 (2012) 2224.

[13] X.Y. Zhang, H.P. Li, X.L. Chui, Y. Lin. Mater. Chem. 20 (2010) 2801

[14] G. Williams, B. Seger, P.V. Kamat. ACS Nano 2 (2008) 1487.

[15] D.C. Marcano, D.V. Kosynkin, J.M. Berlin, A. Sinitskii, Z. Sun, A. Slesarev, L.B. Alemany, W. Lu, J.M. Tour. ACS Nano 4 (2010) 4806.

[16] C. Chen, M. Long, M. Xia, C. Zhang, W. Cain. Nano Res. Lett. 7 (2012) 1.

[17] G. Zhu, T. Xu, T. Lv, L.K. Pan, Q.F. Zhao, Z. Sun. J. Electroanal. Chem. 650 (2011) 248

[18] T.G. Xu, L.W. Zhang, H.Y. Cheng, Y.F. Zhu. Appl. Catal. B: Environ. 101 (2011) 382. 